\title{
Dual pairs of type II string compactification
}

\section{Citation}

Sen, Ashoke, and Cumrun Vafa. 1995. "Dual Pairs of Type II String Compactification." Nuclear Physics B 455 (1-2): 165-87. https://doi.org/10.1016/0550-3213(95)00498-h.

\section{Permanent link}

http://nrs.harvard.edu/urn-3:HUL.InstRepos:41385060

\section{Terms of Use}

This article was downloaded from Harvard University's DASH repository, and is made available under the terms and conditions applicable to Other Posted Material, as set forth at http:// nrs.harvard.edu/urn-3:HUL.InstRepos:dash.current.terms-of-use\#LAA

\section{Share Your Story}

The Harvard community has made this article openly available.

Please share how this access benefits you. Submit a story.

Accessibility 
HUTP-95/A028

TIFR/TH/95-41

hep-th/9508064

\title{
Dual Pairs of Type II String Compactification
}

\author{
Ashoke Sen \\ Tata Institute of Fundamental Research \\ Homi Bhabha Road, Bombay 400005, INDIA \\ and \\ Cumrun Vafa \\ Lyman Laboratory of Physics \\ Harvard University \\ Cambridge, MA 02138, USA
}

\begin{abstract}
Using a $U$-duality symmetry of type II compactification on $T^{4}$ represented by triality action on the $T$-duality group, and applying the adiabatic argument we construct dual pairs of type II compactifications in lower dimensions. The simplicity of this construction makes it an ideal set up for testing various conjectures about string dualities. In some of these models the type II string has a perturbative nonabelian gauge symmetry. Examples include models with $N=2,4,6$ supersymmetry in four dimensions. There are also self-dual (in the sense of $S-T$ exchange symmetric) $N=2$ and $N=6$ examples. A generalization of the adiabatic argument can be used to construct dual pairs of models with $N=1$ supersymmetry.
\end{abstract}




\section{Introduction}

Recently there has been growing evidence for the existence of many different types of duality symmetries in string theory [1]-29]. The existence of duality symmetries has proven to be a powerful tool in extracting exact results about the vacuum structure of string compactifications.

One of the best understood duality symmetries is the conjectured duality between the type IIA compactification on $K 3$ and heterotic compactification on $T^{4}$ [9, 10] for which a lot of evidence has recently emerged [13, 14, 15, 16]. This duality has itself led to other dualities in lower dimensions. In particular upon further toroidal compactification to 4 dimensions, this stringstring duality leads 11, 13 to the strong/weak coupling duality of the $N=4$ supersymmetric string theory in 4 dimensions which had been conjectured to be a stringy generalization[回] [8] of the Olive-Montonen duality [30, 31, 32, 33, 34, 35] in $N=4$ Yang-Mills theory. The mechanism for this reduction is that the $S$-duality of one theory gets mapped to the $T$-duality for the dual theory. If we assume that the $T$-duality is not modified quantum mechanically, we are led to the $S$-duality for the dual theory. One can also seek type II-heterotic dualities in theories with lower number of supersymmetries in four dimensions and some examples of this have been found with $N=1,2$ supersymmetry [18, 19, 27, 29.

As far as string theories with $N=4$ supersymmetry are concerned, there are more ways to get them. On the heterotic side, we can consider asymmetric orbifolds where, roughly speaking, the right-movers are compactified on the six torus and left movers live on an orbifold 36, 37, 38]. At least some of these models have type II dual both in six dimensions 25 and in lower dimensions [39, 27]. The existence of a dual in this class of models, by the same mechanism mentioned above, explains the strong/weak duality of the corresponding heterotic theory. We can get $N=4$ theories in different ways also on the type II side. We can consider compactifications on manifolds which have $S U(2)$ holonomy but which are not $K 3 \times T^{2}$. We can also consider compactifications of type II in which all the 4 spacetime supersymmetries are right-moving, with the left-moving ones being projected out by the symmetry $(-1)^{F_{L}}$ (accompanied by some other symmetries). In fact these models had been previously constructed as a way to obtain non-abelian gauge theories directly from perturbative type II strings [40, 41, 42, 43, 44]. (We can also consider orientifolding the toroidal type II compactifications 
which in many cases is likely to be equivalent to the $(-1)^{F_{L}}$ construction just discussed [27].)

The question arises as to how the $S$-duality of the alternative $N=4$ models coming from these two type II compactifications can be explained. This was the motivation for the present paper, and as we will see, the two alternative $N=4$ type II compactifications will be found to be dual to one another. The basic idea is to start from the conjectured type II $U$-duality in six dimensions 9 and using the adiabatic argument of ref. 27 construct type II dual pairs with fewer supersymmetries in lower dimensions.

As a by-product we are able to construct many more dual pairs in $\mathrm{d}=4$, and in particular with $N=1,2$ supersymmetry. (Construction of dual pairs with $N=1$ supersymmetry requires a generalization of the adiabatic argument, which will be explained in section 4.) The construction of dual type II pairs proceeds in a much simpler way than the corresponding ones starting from the type IIA-heterotic duality in six dimensions. The main reason for this is that for the type IIA-heterotic duality, given the complexity of the conformal theory associated with $K 3$ compactifications, it is rather difficult to find the $K 3$ conformal theory dual to a given heterotic theory (and symmetries being modded out are often not geometrical). For the $T^{4}$ compactification of type II, which is what we are considering, the situation is much simpler and we can easily translate the geometrical symmetry from one toroidal compactification to another, with an explicit map. We can thus produce a large number of dual pairs and this provides a useful laboratory for testing under what operations the duality continues to hold.

The organization of this paper is as follows: In section 2 we review the $U$-duality symmetry of type II string compactified on $T^{4}$. In particular we identify an element $\bar{\Omega}_{0}$ of order 2 in this group and show how it conjugates the 8 dimensional holonomy group (consisting of four left- and four rightmover holonomies) according to $S O(4,4)$ triality. In section 3 we discuss how one may use adiabatic argument and the type II-type II six-dimensional duality given by $\bar{\Omega}_{0}$ to construct models with fewer supersymmetry in lower dimensions. In section 4 we construct a number of such examples, including some with $N=6,4,2$ supersymmetries in four dimensions. We also discuss a plausible $N=1$ dual pair, though it cannot be fully justified by the adiabatic argument. Among the examples constructed a notable one is an $N=2$ model in four dimenions which is self-dual (in the sense that there is an $S-T$ exchange symmetry). 


\section{Type II String Compactified on $T^{4}$}

We begin our discussion in this section by listing the massless fields that appear in the low energy effective action describing type II string theory compactified on a four dimensional torus, and the symmetries of this supergravity theory. Note that toroidal compactifications of type IIA and type IIB are equivalent. For definiteness we will consider type IIA compactifications. The scalar fields in this theory are the dilaton $\Phi$, an $8 \times 8$ matrix valued scalar field $M$ parametrizing the coset $O(4,4) /(O(4) \times O(4))$ corresponding to the moduli associated with $T^{4}$, and a set of 8 scalar fields $\psi^{(\alpha)}(1 \leq \alpha \leq 8)$ associated with the internal components $A_{m}^{[10]}$ and $C_{m n p}^{[10]}(6 \leq m, n, p \leq 9)$ of the ten dimensional bosonic fields arising in the Ramond-Ramond (RR) sector of the theory. $M$ is positive definte, and satisfies the constraints,

$$
M L M^{T}=L, \quad M^{T}=M,
$$

where

$$
L=\left(\begin{array}{cc}
-I_{4} & \\
& I_{4}
\end{array}\right)
$$

$I_{n}$ denotes $n \times n$ identity matrix. The vector fields in this theory consist of $\mathrm{U}(1)$ gauge fields $A_{\mu}^{(a)}(0 \leq \mu \leq 5,1 \leq a \leq 8)$ arising from the components of the ten dimensional metric and anti-symmetric tensor fields $G_{m \mu}^{[10]}$ and $B_{m \mu}^{[10]}$ respectively, and eight $\mathrm{U}(1)$ gauge fields $K_{\mu}^{\left(\alpha^{\prime}\right)}\left(1 \leq \alpha^{\prime} \leq 8\right)$ in the RR sector arising from the components $C_{m n \mu}^{[10]}, A_{\mu}^{[10]}$, and the dual of $C_{\mu \nu \rho}^{[10]}$. This theory also has a symmetric tensor field $g_{\mu \nu}$ denoting the canonical Einstein metric, an antisymmetric tensor field $B_{\mu \nu}$ arising in the Neveu-Schwarz (NS) sector, and four more anti-symmetric tensor fields constructed from the components $C_{m \mu \nu}^{[10]}$. We shall find it convenient to arrange the four field strengths associated with these anti-symmetric tensor fields into eight (anti-)self-dual field strengths $D_{\mu \nu \rho}^{(\alpha)}(1 \leq \alpha \leq 8)$ satisfying

$$
\widetilde{D}_{\mu \nu \rho}^{(\alpha)}=\left(R_{s}(M L)\right)_{\alpha \beta} D_{\mu \nu \rho}^{(\beta)},
$$

where $\widetilde{D}_{\mu \nu \rho}$ denotes the dual of $D_{\mu \nu \rho}$ in six dimensions, and for any $\operatorname{SO}(4,4)$ matrix $\Omega, R_{s}(\Omega)$ and $R_{c}(\Omega)$ denote the two inequivalent spinor representations of $\mathrm{SO}(4,4)$. We shall also denote by $H_{\mu \nu \rho}$ the field strength associated with $B_{\mu \nu}$, and by $\widetilde{H}_{\mu \nu \rho}$ its dual in six dimensions. 
The action for the resulting $N=4$ supergravity theory in six dimensions is invariant under an $\mathrm{SO}(4,4)$ symmetry generated by an $8 \times 8$ matrix $\Omega$ satisfying

$$
\Omega L \Omega^{T}=L, \quad \operatorname{det} \Omega=1 .
$$

The various fields introduced above transform under this symmetry as,

$$
\begin{aligned}
M & \rightarrow \Omega M \Omega^{T}, \\
\psi^{(\alpha)} & \rightarrow\left(R_{s}(\Omega)\right)_{\alpha \beta} \psi^{(\beta)}, \\
D_{\mu \nu \rho}^{(\alpha)} & \rightarrow\left(R_{s}(\Omega)\right)_{\alpha \beta} D_{\mu \nu \rho}^{(\beta)}, \\
A_{\mu}^{(a)} & \rightarrow \Omega_{a b} A_{\mu}^{(b)}, \\
K_{\mu}^{\left(\alpha^{\prime}\right)} & \rightarrow\left(R_{c}(\Omega)\right)_{\alpha^{\prime} \beta^{\prime}} K_{\mu}^{\left(\beta^{\prime}\right)} .
\end{aligned}
$$

$g, H, \widetilde{H}$ and $\Phi$ are invariant. Due to triality, $R_{s}(\Omega)$ and $R_{c}(\Omega)$ themselves can be regarded as $\mathrm{SO}(4,4)$ matrices. In other words, they satisfy the relations

$$
\begin{aligned}
& R_{s}(\Omega) L R_{s}(\Omega)^{T}=L \\
& R_{c}(\Omega) L R_{c}(\Omega)^{T}=L .
\end{aligned}
$$

In general the maps $R_{c}(\Omega)$ and $R_{s}(\Omega)$ are ambiguous, since they depend on the choice of the basis of the spinor representation. We shall use this freedom to choose

$$
R_{c}(\Omega)=R_{s}^{-1}\left(\Omega_{0} R_{s}(\Omega) \Omega_{0}^{-1}\right),
$$

where $\Omega_{0}$ represents the parity transformation matrix

$$
\Omega_{0}=\left(\begin{array}{ccc}
I_{2} & & \\
& \sigma_{3} & \\
& & I_{4}
\end{array}\right) .
$$

It will be useful for us to study the explicit form of the maps $R_{s}(\Omega)$ and $R_{c}(\Omega)$ in some detail. For this, let us consider the $\mathrm{SO}(4,4)$ transformation $\Omega$ to be of the form:

$$
\Omega=\left(\begin{array}{cccc}
\omega\left(\theta_{L}\right) & & & \\
& \omega\left(\phi_{L}\right) & & \\
& & \omega\left(\theta_{R}\right) & \\
& & & \omega\left(\phi_{R}\right)
\end{array}\right),
$$


where

$$
\omega(\theta)=\left(\begin{array}{cc}
\cos \theta & \sin \theta \\
-\sin \theta & \cos \theta
\end{array}\right) .
$$

We shall use the shorthand notation $\left(\theta_{L}, \phi_{L}, \theta_{R}, \phi_{R}\right)$ to denote such a matrix. For such an $\Omega$, the map $R_{s}(\Omega)$ takes the simple form:

$$
R_{s}(\Omega)=\left(\theta_{L}^{\prime \prime}, \phi_{L}^{\prime \prime}, \theta_{R}^{\prime \prime}, \phi_{R}^{\prime \prime}\right),
$$

where,

$$
\left(\begin{array}{l}
\theta_{L}^{\prime \prime} \\
\phi_{L}^{\prime \prime} \\
\theta_{R}^{\prime \prime} \\
\phi_{R}^{\prime \prime}
\end{array}\right)=A_{s}\left(\begin{array}{l}
\theta_{L} \\
\phi_{L} \\
\theta_{R} \\
\phi_{R}
\end{array}\right), \quad A_{s}=\left(\begin{array}{cccc}
\frac{1}{2} & \frac{1}{2} & \frac{1}{2} & -\frac{1}{2} \\
\frac{1}{2} & \frac{1}{2} & -\frac{1}{2} & \frac{1}{2} \\
\frac{1}{2} & -\frac{1}{2} & \frac{1}{2} & \frac{1}{2} \\
-\frac{1}{2} & \frac{1}{2} & \frac{1}{2} & \frac{1}{2}
\end{array}\right) .
$$

Eq.(2.7) now gives

$$
R_{c}(\Omega)=\left(\theta_{L}^{\prime}, \phi_{L}^{\prime}, \theta_{R}^{\prime}, \phi_{R}^{\prime}\right),
$$

for $\Omega$ of the form (2.9), where,

$$
\left(\begin{array}{c}
\theta_{L}^{\prime} \\
\phi_{L}^{\prime} \\
\theta_{R}^{\prime} \\
\phi_{R}^{\prime}
\end{array}\right)=A_{c}\left(\begin{array}{c}
\theta_{L} \\
\phi_{L} \\
\theta_{R} \\
\phi_{R}
\end{array}\right), \quad A_{c}=\left(\begin{array}{cccc}
\frac{1}{2} & -\frac{1}{2} & \frac{1}{2} & -\frac{1}{2} \\
-\frac{1}{2} & \frac{1}{2} & \frac{1}{2} & -\frac{1}{2} \\
\frac{1}{2} & \frac{1}{2} & \frac{1}{2} & \frac{1}{2} \\
-\frac{1}{2} & -\frac{1}{2} & \frac{1}{2} & \frac{1}{2}
\end{array}\right) .
$$

From eqs.(2.9)-(2.14) we get

$$
R_{s}(L)=-L, \quad R_{s}(-L)=L, \quad R_{c}(L)=L .
$$

Using eqs.(2.6) and (2.15) we see that for a general $\Omega \in S O(4,4)$ (not necessarily of the form (2.90),

$$
R_{s}\left(\Omega^{T}\right)=\left(R_{s}(\Omega)\right)^{T}, \quad R_{c}\left(\Omega^{T}\right)=\left(R_{c}(\Omega)\right)^{T} .
$$

This implies, in particular, that if $\Omega$ is symmetric, then so are $R_{s}(\Omega)$ and $R_{c}(\Omega)$. Also if $\Omega \in O(4)_{L} \times O(4)_{R}$ then $R_{s}(\Omega), R_{c}(\Omega) \in O(4)_{L} \times O(4)_{R}$. These properties will be useful for us later.

The equations of motion of the theory in fact have a larger symmetry belonging to the group $S O(5,5)[46,47,48$. This group is generated by $5 \times 5$ matrices $\bar{\Omega}$ satisfying,

$$
\bar{\Omega} \bar{L} \bar{\Omega}^{T}=\bar{L}, \quad \operatorname{det} \bar{\Omega}=1,
$$


where,

$$
\bar{L}=\left(\begin{array}{ll}
\sigma_{1} & \\
& L
\end{array}\right), \quad \sigma_{1}=\left(\begin{array}{ll} 
& 1 \\
1 &
\end{array}\right) .
$$

The $\mathrm{SO}(4,4)$ group discussed before is embedded in $\mathrm{SO}(5,5)$ as

$$
\bar{\Omega}=\left(\begin{array}{ll}
I_{2} & \\
& R_{s}(\Omega)
\end{array}\right) .
$$

To see how the full $\mathrm{SO}(5,5)$ symmetry acts on the various fields, it is convenient to introduce a new matrix valued field

$$
\bar{M}=\left(\begin{array}{ccc}
e^{2 \Phi} & -\frac{1}{2} e^{2 \Phi} \psi^{T} L \psi & -e^{2 \Phi} \psi^{T} \\
-\frac{1}{2} e^{2 \Phi} \psi^{T} L \psi & e^{-2 \Phi}+\psi^{T} L R_{s}(M) L \psi & \psi^{T} L R_{s}(M) \\
& +\frac{1}{4} e^{2 \Phi}\left(\psi^{T} L \psi\right)^{2} & +\frac{1}{2} e^{2 \Phi} \psi^{T}\left(\psi^{T} L \psi\right) \\
-e^{2 \Phi} \psi & R_{s}(M) L \psi+\frac{1}{2} e^{2 \Phi} \psi\left(\psi^{T} L \psi\right) & R_{s}(M)+e^{2 \Phi} \psi \psi^{T}
\end{array}\right),
$$

satisfying,

$$
\bar{M} \bar{L} \bar{M}=\bar{L}, \quad \bar{M}^{T}=\bar{M} .
$$

We also define,

$$
P_{\mu}=\left(\begin{array}{c}
\vec{K}_{\mu} \\
\vec{A}_{\mu}
\end{array}\right)+\mathcal{O}(\psi),
$$

and,

$$
Q_{\mu \nu \rho}=\left(\begin{array}{c}
H_{\mu \nu \rho} \\
e^{-2 \Phi} \stackrel{\sim}{H}_{\mu \nu \rho} \\
\vec{D}_{\mu \nu \rho}
\end{array}\right)+\mathcal{O}(\psi),
$$

satisfying the self-duality condition

$$
\widetilde{Q}_{\mu \nu \rho}=\bar{M} \bar{L} Q_{\mu \nu \rho} .
$$

$\mathcal{O}(\psi)$ terms in eqs.(2.22), (2.23) reflect the fact that the field combinations $P_{\mu}$ and $Q_{\mu \nu \rho}$ which transform covariantly under $\mathrm{SO}(5,5)$ have order $\psi^{(\alpha)}$ terms in them which we have not written down explicitly. Since we shall focus on backgrounds with vanishing $\psi^{(\alpha)}$, we shall not need to know the explicit form of these terms. 
If $R_{S}(\bar{\Omega})$ denotes the $16 \times 16$ matrix corresponding to the spinor representation of $S O(5,5)$, then the transformation laws of various fields under the $\mathrm{SO}(5,5)$ transformation is given as follows:

$$
\begin{aligned}
g_{\mu \nu} & \rightarrow g_{\mu \nu}, \\
\bar{M} & \rightarrow \bar{\Omega} \bar{M} \bar{\Omega}^{T}, \\
P_{\mu} & \rightarrow R_{S}(\bar{\Omega}) P_{\mu}, \\
Q_{\mu \nu \rho} & \rightarrow \bar{\Omega} Q_{\mu \nu \rho} .
\end{aligned}
$$

Using eq.(2.19) and the well known decomposition property of the spinor representation of $\mathrm{SO}(5,5)$ under $\mathrm{SO}(4,4)$ :

$$
R_{S}(\bar{\Omega})=\left(\begin{array}{cc}
R_{c}(\Omega) & \\
& \Omega
\end{array}\right), \quad \text { for } \quad \bar{\Omega}=\left(\begin{array}{ll}
I_{2} & \\
& R_{s}(\Omega)
\end{array}\right),
$$

one can verify that the $\mathrm{SO}(5,5)$ transformation laws given in eq.(2.25) are compatible with the $\mathrm{SO}(4,4)$ transformation laws given in eq.(2.5).

An $\mathrm{SO}(4,4 ; \mathrm{Z})$ subgroup of $\mathrm{SO}(4,4)$ is known to be a symmetry of the type IIA string theory compactified on $T^{4}$ order by order in perturbation theory, and is known as the $T$-duality group of this theory. For definiteness, we shall always choose our reference $T^{4}$ to be the one consisting of four mutually orthogonal cycles with each cycle at the self-dual radius, and no background $B_{m n}$ field. If $\Gamma_{0}^{(4,4)}$ denotes the lattice of integers labelling the momenta and winding numbers on such a torus, then $\mathrm{SO}(4,4 ; \mathrm{Z})$ is the subgroup of $\mathrm{SO}(4,4)$ which leaves this lattice invariant.

An $\mathrm{SO}(5,5 ; \mathrm{Z})$ subgroup of the $\mathrm{SO}(5,5)$ group has been conjectured to be an exact non-perturbative symmetry of this string theory[9]. A particular element of this $\mathrm{SO}(5,5 ; \mathrm{Z})$ group, which will play an important role in our analysis, is

$$
\bar{\Omega}_{0}=\left(\begin{array}{cc}
\sigma_{1} & \\
& \Omega_{0}
\end{array}\right), \quad \Omega_{0}=\left(\begin{array}{ccc}
I_{2} & & \\
& \sigma_{3} & \\
& & I_{4}
\end{array}\right) .
$$

We shall conclude this section by listing some of the important properties of this $\mathrm{SO}(5,5 ; \mathrm{Z})$ transformation $\bar{\Omega}_{0}$ :

- From eqs.(2.19)-(2.25) we see that for $\psi^{(\alpha)}=0, \bar{\Omega}_{0}$ induces the transformation

$$
\Phi \rightarrow-\Phi, \quad H_{\mu \nu \rho} \rightarrow e^{-2 \Phi} \widetilde{H}_{\mu \nu \rho}
$$


Thus the transformation induced by $\bar{\Omega}_{0}$ acts on the $\left\{\Phi, B_{\mu \nu}\right\}$ combination in the same way that string-string duality transformation acts on these fields [11, 13].

- $\bar{\Omega}_{0}$ conjugates an element of $\mathrm{SO}(4,4)$ to another element of $\mathrm{SO}(4,4)$ :

$$
\bar{\Omega}_{0}\left(\begin{array}{cc}
I_{2} & \\
& R_{s}(\Omega)
\end{array}\right) \bar{\Omega}_{0}^{-1}=\left(\begin{array}{cc}
I_{2} & \\
& \Omega_{0} R_{s}(\Omega) \Omega_{0}^{-1}
\end{array}\right)=\left(\begin{array}{ll}
I_{2} & \\
& R_{s}\left(R_{c}(\Omega)\right)
\end{array}\right),
$$

using eqs.(2.7). Thus the net effect of conjugating an $S O(4,4)$ transformation $\Omega$ by the $S O(5,5)$ transformation $\bar{\Omega}_{0}$ is another $S O(4,4)$ transformation $R_{c}(\Omega)$.

- Since conjugation by $\bar{\Omega}_{0}$ exchanges the $\mathrm{SO}(4,4)$ matrices $\Omega$ and $R_{c}(\Omega)$, we see from (2.5) that the action of $\bar{\Omega}_{0}$ exchanges the NS sector gauge fields $\left\{A_{\mu}^{(a)}\right\}$ with the RR sector gauge fields $\left\{K_{\mu}^{\left(\alpha^{\prime}\right)}\right\}$. By choosing appropriate basis to describe the set of fields $\left\{A_{\mu}^{(a)}\right\}$ and $\left\{K_{\mu}^{\left(\alpha^{\prime}\right)}\right\}$ we can have (see eq.(2.25)):

$$
R_{S}\left(\bar{\Omega}_{0}\right)=\left(\begin{array}{cc}
0 & I_{8} \\
-I_{8} & 0
\end{array}\right)
$$

The origin of the minus sign on the lower left hand corner of this matrix is the fact that $\bar{\Omega}_{0}$ represents a rotation by $\pi$ inside $S O(5,5)$. Thus we expect $R_{S}\left(\bar{\Omega}_{0}^{2}\right)$ to be represented by $-I_{16}$.

- A realization of the duality transformation $\bar{\Omega}_{0}$ in terms of the more familiar duality transformations may be given as follows. Let us consider the theory as a compactification of the type IIB theory. This theory has an $\mathrm{SL}(2, \mathrm{Z})$ S-duality symmetry in ten dimensions; let us denote by $\sigma$ the element of this $\mathrm{SL}(2, \mathrm{Z})$ group that inverts the ten dimensional $S$ field. After we compactify the theory on $T^{4}$, it also has various $T$-duality symmetries. Let us denote by $\tau_{m n}$ the duality transformation that takes the $T$-modulus associated with the $m-n$ plane $(6 \leq m, n \leq 9)$ to its inverse. Then $\bar{\Omega}_{0}$ can be regarded as the following combination of these duality transformations:

$$
\sigma \cdot \tau_{67} \cdot \tau_{89} \cdot \sigma^{-1}
$$


- The full U-duality group is enhanced if we compactify the theory on one or more circles, but $\bar{\Omega}_{0}$ remains a valid U-duality transformation in these theories.

\section{Method for Constructing Dual Pairs of The- ories}

We shall begin this section by outlining the method for constructing new pairs of theories that are dual to each other. In view of potential generalizations to other cases, we will first discuss the method in more generality and then specialize to the case at hand. Suppose we consider a theory with $U$-duality group $G$. This of course contains the $T$-duality group $H \subset G$ which is the symmetry of the perturbative string theory. Suppose $h, h^{\prime} \in H$ are elements of finite order $n$ conjugate to one-another in $G$ but not in $H$, i.e. $h^{\prime}=g h g^{-1}$ where $g \in G$ but $g \notin H$. By general arguments there is a subspace $\mathcal{M}\left(\mathcal{M}^{\prime}\right)$ of moduli space of compactifications left invariant by $h\left(h^{\prime}\right)$, which implies that the corresponding perturbative string has $h\left(h^{\prime}\right)$ as a symmetry. Note that $g \cdot \mathcal{M}=\mathcal{M}^{\prime}$. Start from a string compactification characterized by a point $m \in \mathcal{M}$. Compactify on a circle, and mod out the theory by a simultaneous action of $h$, and a translation of $1 / n$ unit along the circle. In other words, as we go a fraction $1 / n$ times around the circle we identify the internal theory with the action of $h$. Consider a dual theory starting from $m^{\prime}=g \cdot m$ on the moduli space. Compactify on a circle where as we go a fraction $1 / n$ times around the circle, we come back to the same conformal theory acted on by $h^{\prime}$. By the adiabatic argument of [27] we expect the two resulting theories to be dual. Moreover this duality cannot be seen perturbatively because $h$ and $h^{\prime}$ are not conjugate in $H$. Note that we can compactify further and mod out by more symmetries to get other dual pairs in lower dimensions, consistent with the adiabatic argument.

A particularly nice case of the above construction is when there exists a special element $g \in G$ such that for all $h \in H, g h g^{-1} \in H$, but that $g \notin H$. If this happens we can use any finite order $h \in H$ to construct dual pairs. This luckily is the case for us.

Let us now specialize to our case corresponding to $T^{4}$ compactification of type IIA strings where $G=S O(5,5 ; Z), H=S O(4,4 ; Z)$ and $g=\bar{\Omega}_{0}$ 
constructed in the previous section. So our strategy is as follows.

- We start with type IIA theory compactified on $T^{4}$ characterized by the moduli $\langle\bar{M}\rangle$ which is invariant under some $\mathrm{SO}(4,4 ; \mathrm{Z})$ transformation $\widehat{\Omega}$. This theory is equivalent to type IIA theory characterized by a modular parameter

$$
\left\langle\bar{M}^{\prime}\right\rangle=\bar{\Omega}_{0}\langle\bar{M}\rangle \bar{\Omega}_{0}^{T}
$$

which is invariant under the $\mathrm{SO}(4,4 ; \mathrm{Z})$ transformation

$$
\widehat{\Omega}^{\prime}=R_{c}(\widehat{\Omega})
$$

according to eq.(2.7).

- Now let us further compactify both the theories on $T^{2}$. If $\widehat{\Omega}$ is of order $n$, then we mod out the first theory by simultaneous action of $\widehat{\Omega}$ and a translation on the first circle of $T^{2}$ equal to $1 / n$ times the radius of the circle. Similarly we mod out the second theory by simultaneous action of the $\mathrm{SO}(4,4 ; \mathrm{Z})$ transformation $\widehat{\Omega}^{\prime}$ and a translation on the first circle of $T^{2}$ equal to $1 / n$ times the radius of the circle. Using the adiabatic argument given in ref.[27] we expect the two resulting theories to be equivalent.

This gives the general method for constructing dual pairs of theories. We now discuss some of the subtleties and special features that arise in this construction.

- The adibatic argument by itself does not rule out extra shifts in the winding direction on the lattice $\Gamma^{(1,1)}$ associated with the first circle. But in the cases we shall discuss this extra shift is forced to vanish in order to have level matching between the left and right moving components of the string states.

- The construction could be carried out in five dimensions, since the operations used in modding out the two theories did not involve the second circle. In fact we can further mod out both the theories by extra $\mathrm{SO}(4,4 ; \mathrm{Z})$ symmetries accompanied by shifts on the second circle to get more examples of dual pairs of theories. The adiabatic argument showing the equivalence of these two theories again applies since we can take the radius of the second circle to be large. 
- Instead of working with the duality transformations $\widehat{\Omega}\left(\widehat{\Omega}^{\prime}\right)$ which represent symmetries of $\langle M\rangle\left(\left\langle M^{\prime}\right\rangle\right)$, it is more convenient to work with the transformations which represent symmetries of the corresponding Narain lattice. To do this, let us decompose $\langle M\rangle$ as

$$
\langle M\rangle=\mathcal{V}^{T}, \quad \mathcal{V} \in S O(4,4)
$$

$\mathcal{V}$ is defined up to an $O(4) \times O(4)$ multiplication from the right. Invariance of $\langle M\rangle$ under $\widehat{\Omega}$ then implies that

$$
\Omega \equiv \mathcal{V}^{-1} \widehat{\Omega} \mathcal{V} \in O(4) \times O(4)
$$

Furthermore, the freedom of multiplying $\mathcal{V}$ from the right by an $O(4) \times$ $O(4)$ matrix translates to a conjugation of $\Omega$ by an $O(4) \times O(4)$ matrix. Using this freedom we can always bring $\Omega$ to the form (2.9). This $O(4) \times O(4)$ matrix $\Omega$ now represents the symmetry of the lattice $\Gamma^{(4,4)} \equiv \mathcal{V}^{-1} \Gamma_{0}^{(4,4)}$ describing the specific compactification. $(O(4) \times O(4)$ conjugation of $\Omega$ reflects the freedom in choosing the axes for describing the lattice.)

Similarly, if we define,

$$
M^{\prime}=\mathcal{V}^{\prime} \mathcal{V}^{\prime T}, \quad \Omega^{\prime}=\mathcal{V}^{\prime-1} \widehat{\Omega}^{\prime} \mathcal{V}^{\prime} \in O(4) \times O(4),
$$

then $\Omega^{\prime}$ corresponds to the symmetry of the Narain lattice $\Gamma^{(4,4) \prime} \equiv$ $\mathcal{V}^{\prime-1} \Gamma_{0}^{(4,4)}$ describing the dual theory. Using eqs.(3.1)-(3.5) and (2.7) we see that

$$
\mathcal{V}^{\prime}=R_{c}(\mathcal{V})
$$

and,

$$
\Omega^{\prime}=R_{c}(\Omega) .
$$

Modding out the theories described by $\langle M\rangle\left(\left\langle M^{\prime}\right\rangle\right)$ by the symmetries $\widehat{\Omega}\left(\widehat{\Omega}^{\prime}\right)$ is equivalent to modding out the corresponding lattices $\Gamma^{(4,4)}$ $\left(\Gamma^{(4,4) \prime}\right)$ by the symmetries $\Omega\left(\Omega^{\prime}\right)$. Since $\Omega$ is of the form $(2.9), \Omega^{\prime}$ can be easily found using eqs.(2.11)-(2.14). For a given lattice $\Gamma^{(4,4)}=$ $\mathcal{V}^{-1} \Gamma_{0}^{(4,4)}$, the lattice $\Gamma^{(4,4) \prime}=\mathcal{V}^{\prime-1} \Gamma_{0}^{(4,4)}$ of the dual theory can be found using eq. 3.6). We have however not given the map $R_{c}(\mathcal{V})$ explicitly for general $\mathcal{V}$ which is not of the form (2.9). 
- Since the relationship between the dilaton and the $B_{\mu \nu}$ fields in the two theories given in eq.(2.28) is identical to the one that describes string-string duality in six dimensions, the resulting four dimensional theories will have their $S$ - and $T$ - moduli exchanged:

$$
S^{\prime}=T, \quad T^{\prime}=S .
$$

- For definiteness, let us choose $T^{2}$ to be the torus with two orthogonal cycles at self-dual radii, and no background $B_{i j}$ field. Let $\Gamma_{0}^{(2,2)}$ denote the lattice associated with this two dimensional torus. In the basis where the metric is of the form $\left(\begin{array}{cc}0 & I_{2} \\ I_{2} & 0\end{array}\right), \Gamma_{0}^{(2,2)}$ is the lattice of integers. (A general lattice $\Gamma^{(2,2)}$ can be obtained from $\Gamma_{0}^{(2,2)}$ by an $O(2,2)$ boost, which corresponds to switching on the background $G_{i j}$ and $B_{i j}$ fields.) In this case the shift on the first circle accompanying the $\mathrm{SO}(4,4 ; \mathrm{Z})$ transformation $\Omega$ or $\Omega^{\prime}$ is represented by a vector

$$
v=\left(\begin{array}{c}
\frac{1}{n} \\
0 \\
0 \\
0
\end{array}\right) \text {. }
$$

Then the duality symmetry group $\mathrm{SO}(2,2 ; \mathrm{Z})$ associated with $T^{2}$ is broken down to a subgroup that preserves the vector $v$ up to lattice translation. (For $\mathrm{N}=2$ or $\mathrm{N}=1$ supersymmetric theories this duality group is further modified due to quantum corrections, but for $\mathrm{N} \geq 4$ supersymmetric compactification we expect this to be the exact duality symmetry group of the theory associated with $T^{2}$.) In order to determine what the surviving duality group is it is convenient to parametrize an $\mathrm{SO}(2,2 ; \mathrm{Z})=\mathrm{SL}(2, \mathrm{Z})_{T} \times \mathrm{SL}(2 ; \mathrm{Z})_{U}$ matrix as

$$
\left(\begin{array}{cccc}
p_{1} p_{2} & p_{1} q_{2} & -q_{1} q_{2} & q_{1} p_{2} \\
p_{1} r_{2} & p_{1} s_{2} & -q_{1} s_{2} & q_{1} r_{2} \\
-r_{1} r_{2} & -r_{1} s_{2} & s_{1} s_{2} & -s_{1} r_{2} \\
r_{1} p_{2} & r_{1} q_{2} & -s_{1} q_{2} & s_{1} p_{2}
\end{array}\right)
$$

where,

$$
\left(\begin{array}{ll}
p_{1} & q_{1} \\
r_{1} & s_{1}
\end{array}\right) \in S L(2, Z)_{T}, \quad\left(\begin{array}{ll}
p_{2} & q_{2} \\
r_{2} & s_{2}
\end{array}\right) \in S L(2, Z)_{U}
$$


From eqs.(3.9) and (3.10) we see that in order to preserve $v$ up to lattice translations we need,

$$
\begin{array}{ll}
p_{1} p_{2}=1 \bmod n, & r_{1} r_{2}=0 \bmod n, \\
p_{1} r_{2}=0 \bmod n, & r_{1} p_{2}=0 \bmod n .
\end{array}
$$

This gives

$$
\left(\begin{array}{ll}
p_{1} & q_{1} \\
r_{1} & s_{1}
\end{array}\right)=\left(\begin{array}{cc}
1 & q_{1} \\
0 & 1
\end{array}\right) \bmod n, \quad\left(\begin{array}{ll}
p_{2} & q_{2} \\
r_{2} & s_{2}
\end{array}\right)=\left(\begin{array}{cc}
1 & q_{2} \\
0 & 1
\end{array}\right) \bmod n
$$

Thus in both the theories $\mathrm{SL}(2, \mathrm{Z})_{T} \times \mathrm{SL}(2, \mathrm{Z})_{U}$ is broken down to $\Gamma_{0}(n) \times$ $\Gamma_{0}(n)$. Since the $S$ and $T$ moduli are exchanged in the two theories, this shows that the S-duality group $\mathrm{SL}(2, \mathrm{Z})_{S}$ is also broken down to $\Gamma_{0}(n)$.

- The full U-duality group of the theory may be found by combining the subgroup of the six dimensional U-duality group that commutes with the T-duality transformation $\widehat{\Omega}$ and the full T-duality group of the four dimensional theory. The element $\widehat{\Omega}$ itself is a symmetry of the quantum theory [49], but it does not have the interpretation of a conventional duality symmetry since it leaves all the moduli fields invariant.

- Finally, we note that after modding out the two theories by appropriate group of transformations, we can switch on the Wilson lines for the background six dimensional gauge fields that survive the projection by $\Omega\left(\Omega^{\prime}\right)$. For NS background fields this will correspond to a deformation of the internal lattice $\Gamma^{(6,6)}$ away from its factorized from $\Gamma^{(4,4)} \times \Gamma^{(2,2)}$ consistent with invariance under $\Omega\left(\Omega^{\prime}\right)$. For RR Wilson lines, there is no such simple geometrical interpretation. Since the $\mathrm{SO}(5,5)$ transformation $\bar{\Omega}_{0}$ maps NS sector gauge fields to RR sector gauge fields and vice versa, we see that a deformation of the lattice $\Gamma^{(6,6)}$ from its factorized form in one theory corresponds to switching on RR Wilson lines in the other theory, and vice versa.

\section{Explicit Examples of Dual Pairs of Models}

In this section we shall construct explicit examples of dual pairs of models following the procedure outlined in the previous section. We will concentrate 
on constructing supersymmetric models in four dimensions, though other dimensions can also be considered. In type II compactifications, the number of spacetime supersymmetries we can obtain from each left- or right-mover Hilbert space is $0,1,2$ or 4 , corresponding to compactifications with left- or right-moving holonomy in $S O(6), S U(3), S U(2), 1$. We shall call a theory with $n$ left moving and $m$ right moving space-time supersymmetry a theory of the $\left(n_{L}, m_{R}\right)$ type. Combining left- and right-moving supersymmetries we can thus obtain any $N=n+m$ between 0 and 8 except $N=7$ (the $N=7$ presumably gets automatically promoted to $N=8$ ) - for some examples of such models see [40, 41, 42, 43, 44]. As far as adiabatic argument is concerned, we need to leave at least one circle invariant, which implies that we can have all the above holonomy groups except $S U(3)$. Thus if we wish to apply the adiabatic argument we will have to restrict to 0,2,4 supersymmetries from each side, which gives us possible total supersymmetries as $N=2,4,6,8$ (where we omit $N=0$ because it is not clear how to make sense of nonsupersymmetric string theories). The $N=8$ theory can be obtained in a unique way, which is continuing toroidal compactification down to four dimensions. $N=6$ can be constructed by decomposing $6=4+2$ and so we use toroidal compactification on one side and $S U(2)$ holonomy on the other side. $N=4$ theories can be constructed in two ways by decomposing $4=2+2$ or $4=4+0$. In the first case we can consider compactifications with $S U(2)$ holonomy for both left- and right-movers and so we can use geometric compactifications. For the latter case we can use $S O(6)$ holonomy (or $(-1)^{F_{L}}$ ) on one side and toroidal compactification on the other. For $N=2$ theories, again there is a unique way of decomposition as $2=2+0$ corresponding to $S U(2)$ holonomy on one side and $S O(6)$ holonomy on the other.

Note that specifying the holonomy we are modding out by does not uniquely specify the action. There are two potential other choices: 1- There may be inequivalent realizations of the same holonomy group action on different lattices. 2-We can also accompany the holonomy action by translations in the internal theory, which when using the duality map discussed in section 2 get mapped to turning on RR Wilson lines in the dual theory. Both of these considerations will be relevant below in connection with constructing the dual to $N=4$ models of ref. 44].

It turns out that the simplest models one can construct with $N=2$ and $N=6$ are self-dual, mirroring the fact that there is a unique way of de- 
composing the supersymmetries between the left- and right-movers. On the other hand, the two classes of $N=4$ models, depending on the decomposition of supersymmetry, are dual to one another. In particular in this way we find the dual for the $N=4$ models of [4], corresponding to the decomposition $4=0+4$, as $S U(2)$ holonomy compactifications corresponding to the decomposition $4=2+2$.

We shall first discuss examples with $N=6,4$ and 2 . To proceed further to other values of $N$ we have to go beyond the adiabatic argument. In order to do this we follow an idea, though not justifiable by adiabatic argument, which we find reasonable. This idea will be explained at the end of this section and some examples with $N=1,2$ supersymmetries will be constructed along that line.

N=6 Example: We choose,

$$
\left(\theta_{L}, \phi_{L}, \theta_{R}, \phi_{R}\right)=(\pi,-\pi, 0,0)
$$

Eq. 2.14) now gives,

$$
\left(\theta_{L}^{\prime}, \phi_{L}^{\prime}, \theta_{R}^{\prime}, \phi_{R}^{\prime}\right)=(\pi,-\pi, 0,0)
$$

Note that the holonomies in the two theories are the same. Also, the lattice with this symmetry is the original lattice $\Gamma_{0}^{(4,4)}$. This gives $\langle M\rangle=\left\langle M^{\prime}\right\rangle=I_{8}$. Thus the model is self-dual.

The associated shifts $v, v^{\prime}$ along one of the circles (say the fourth direction) by half the periodicity is represented by the lattice vector

$$
v=v^{\prime}=\left(\begin{array}{c}
\frac{1}{2} \\
0 \\
0 \\
0
\end{array}\right)
$$

on $\Gamma_{0}^{(2,2)}$. The adiabatic argument by itself does not rule out the possibility of adding a vector $\left(0,0, \frac{1}{2}, 0\right)$ to $v$ and /or $v^{\prime}$, but the left-right level matching condition determines both $v$ and $v^{\prime}$ uniquely to be given by eq.(4.3).

The transformation $\Omega$ given in eq. (4.1) corresponds to an $S U(2)$ holonomy on the left-movers and trivial holonomy on the right-movers, giving us an $N=$ 6 model. The self-duality of the model reflects the fact that the $\mathrm{SO}(5,5 ; \mathrm{Z})$ element $\bar{\Omega}_{0}$ commutes with the $T$-duality transformation generated by $\Omega$, 
and hence is an element of the U-duality group of the resulting theory. The spectrum of massless states in this theory can be found as follows. First we note that under the $Z_{2}$ subgroup of the $\mathrm{SO}(4,4 ; \mathrm{Z})$ group generated by (4.1), the different $\mathrm{SO}(4,4)$ representations decompose as,

$$
\begin{aligned}
& 8_{v}=4(+) \oplus 4(-) \\
& 8_{s}=4(+) \oplus 4(-) \\
& 8_{c}=4(+) \oplus 4(-) .
\end{aligned}
$$

Thus we get four vector fields from $A_{\bar{\mu}}^{(a)}(0 \leq \bar{\mu} \leq 3)$, four from $K_{\bar{\mu}}^{\left(\alpha^{\prime}\right)}$, four $(=4 \times 2 / 2)$ from the field strengths $D_{i \bar{\mu} \bar{\nu}}^{(\alpha)}(4 \leq i \leq 5)$ and four more vector fields from $G_{i \bar{\mu}}$ and $B_{i \bar{\mu}}$ (since $D$ is self-dual, two $D_{i \bar{\mu} \bar{\nu}}^{(\alpha)}$ make one unconstrained vector field). This gives a total of 16 vectors which is the right number for the $\mathrm{N}=6$ supergravity theory in four dimensions 46]. Similar counting shows that we get the right number (30) of scalar fields as well.

As noted in [27] the $U$-duality group for $N=4,5,6$ will depend on the precise choice of compactification. In this example, part of the T-duality group associated with the torus $T^{2}$ is given by $\Gamma_{0}(2)_{T} \times \Gamma_{0}(2)_{U}$. Since this model is self-dual, it has an $\mathrm{S}-\mathrm{T}$ exchange symmetry which we shall call $\left(Z_{2}\right)_{S-T}$. This also shows that the S-duality group is $\Gamma_{0}(2)_{S}$. The full U-duality group $G$ can be found following the procedure indicated in the previous section.

The moduli space of the theory locally has the structure $\mathrm{SO}^{*}(12) / \mathrm{U}(6)$. Thus the global structure of the moduli space is given by $G \backslash S O^{*}(12) / U(6)$. $\mathbf{N}=4$ Example A: We now turn to constructing $N=4$ dual pairs. We choose,

$$
\left(\theta_{L}, \phi_{L}, \theta_{R}, \phi_{R}\right)=(2 \pi, 0,0,0)
$$

Eq.(2.14) now gives,

$$
\left(\theta_{L}^{\prime}, \phi_{L}^{\prime}, \theta_{R}^{\prime}, \phi_{R}^{\prime}\right)=(\pi,-\pi, \pi,-\pi)
$$

The transformation $\Omega$ given in eq.(4.5) corresponds to $(-1)^{F_{L}}$ since it involves a rotation by $2 \pi$ in the $6_{L}-7_{L}$ plane. On the other hand, the transformation $\Omega^{\prime}$ corresponds to an inversion of $T^{4}$, i.e. an inversion of the lattice $\Gamma^{(4,4) \prime}$. The associated shifts $\left(v, v^{\prime}\right)$ along one of the circles (say the fourth direction) 
by half the periodicity is represented by the lattice vector

$$
v=v^{\prime}=\left(\begin{array}{c}
\frac{1}{2} \\
0 \\
0 \\
0
\end{array}\right)
$$

on $\Gamma_{0}^{(2,2)}$.

We now turn to a comparison of the two theories.

- In the first theory (obtained after modding out by $\Omega$ together with the lattice translation $v$ ) the space-time supersymmetry generators on the left are all broken, whereas those on the right are all intact. Thus this theory has $\left(0_{L}, 4_{R}\right)$ supersymmetry in four dimensions. This gives a total of $\mathrm{N}=4$ supersymmetry. On the other hand, the second theory has $\left(2_{L}, 2_{R}\right)$ space-time supersymmetry, which again gives $\mathrm{N}=4$ supersymmetry in four dimensions. Note that the $\left(2_{L}, 2_{R}\right)$ theory is a geometric compactification of strings on an $S U(2)$ holonomy manifold which is not $K 3 \times T^{2}$.

- In the first theory all states that come from the Ramond sector on the left are projected out. This, in particular, means that there are no states from the RR sector. Thus the gauge fields that survive the projection are $A_{\bar{\mu}}^{(a)}, G_{i \bar{\mu}}$ and $B_{i \bar{\mu}}$. This gives a total of 12 gauge fields. On the other hand, in the second theory, the gauge fields $A_{\bar{\mu}}^{\prime(a)}$, which originate from the internal components $G_{m \bar{\mu}}^{[10]}, B_{m \bar{\mu}}^{[10]}(6 \leq m \leq 9)$, as well as the gauge fields which originate from the components $C_{m i \bar{\mu}}^{[10]}$, get projected out since they are odd under the inversion of the four torus, but the gauge fields $K_{\bar{\mu}}^{\prime\left(\alpha^{\prime}\right)}, G_{i \bar{\mu}}^{\prime}$ and $B_{i \bar{\mu}}^{\prime}$ survive. This again gives a total of 12 gauge fields.

- Since in the $N=4$ supergravity theories the structure of the low energy effective action is completely determined by the number of $\mathrm{U}(1)$ gauge fields, the two theories have identical low energy effective action. In particular, the local structure of the moduli space in both the theories is given by $(S L(2, R) / U(1)) \times(O(6,6) / O(6) \times O(6))$. In the first theory the $S L(2, R) / U(1)$ component of the moduli space is parametrized by 
the axion-dilaton field $S$, whereas the moduli $T$ and $U$ of $T^{2}$, the internal moduli of $T^{4}$, and the Wilson lines of the NS sector gauge fields parametrize the $(O(6,6) / O(6) \times O(6))$ component of the moduli space. On the other hand, in the second theory the $S L(2, R) / U(1)$ component of the moduli space is parametrized by the modulus $T^{\prime}$ of $T^{2}$. The axion-dilaton field $S^{\prime}$, the modulus $U^{\prime}$ of $T^{2}$, the internal moduli of $T^{4}$, and the RR Wilson lines parametrize the coset $(O(6,6) / O(6) \times O(6))$. This is consistent with our previous assertion that the field $S$ should be identified with $T^{\prime}$, and that $T$ should be identified with $S^{\prime}$.

Using the duality between these two theories we can get information about the duality symmetry group of the individual theories. In particular, according to the argument given before, in both the theories the duality groups acting on the $\mathrm{S}, \mathrm{T}$ and $\mathrm{U}$ fields are given by $\Gamma_{0}(2)$. Thus globally the moduli space has the structure

$$
\left(\Gamma_{0}(2) \backslash S L(2, R) / U(1)\right) \times(G \backslash O(6,6) / O(6) \times O(6)),
$$

where

$$
G \cap O(2,2)=\Gamma_{0}(2) \times \Gamma_{0}(2) .
$$

Finally we turn to the study of enhanced gauge symmetries. In the first theory, by adjusting $G_{i j}, B_{i j}$ (i.e. the moduli $T$ and $U$ ), or equivalently, by performing an $\mathrm{O}(2,2)$ boost with the matrix

$$
\mathcal{V}^{-1}=\frac{1}{\sqrt{2}}\left(\begin{array}{cccc}
0 & 0 & 0 & 1 \\
2 & 0 & 0 & 1 \\
2 & 2 & -1 & 1 \\
0 & 0 & 1 & -1
\end{array}\right)
$$

we can bring the lattice $\Gamma_{0}^{(2,2)}$ to the form $\Gamma^{(2,2)}\left(D_{2}\right)$ where:

$$
\Gamma^{(l, l)}\left(D_{l}\right)=\left\{\frac{1}{\sqrt{2}}\left(\begin{array}{c}
m_{1} \\
\cdot \\
\cdot \\
m_{l} \\
n_{1} \\
\cdot \\
\cdot \\
n_{l}
\end{array}\right)\right\}, \quad m_{i}, n_{i} \in Z, \quad \sum_{i=1}^{l} m_{i}, \sum_{i=1}^{l} n_{i} \in 2 Z,
$$




$$
m_{i}+n_{i}+m_{j}+n_{j} \in 2 Z \forall(i, j) .
$$

The same boost brings the shift vector $v$ to the form:

$$
v=\frac{1}{\sqrt{2}}\left(\begin{array}{l}
1 \\
0 \\
1 \\
0
\end{array}\right),
$$

up to a lattice translation. According to the analysis of ref. 444 this theory has an enhanced gauge symmetry $(S U(2))^{2}$. Then the equivalence of the two theories implies that the second theory also has an enhanced gauge symmetry for these values of the moduli. The map between the fields in the two theories shows that the massless charged vector particles in the second theory carry magnetic type $B_{i \bar{\mu}}^{\prime}$ charge and hence are non-perturbative states in the spectrum. Furthermore, in order to reach the enhanced symmetry point, we need to adjust the moduli $U^{\prime}$ and $S^{\prime}$ in the second theory. Thus the symmetry enhancement is a non-perturbative phenomenon in the second theory.

We can get further symmetry enhancement in the first theory by adjusting the moduli of $T^{4}$ and $T^{2}$, as well as the Wilson lines associated with the gauge field components $A_{i}^{(a)}$. This is equivalent to an $\mathrm{O}(6,6)$ boost. With the help of this boost we can bring the lattice to the form $\Gamma^{(6,6)}\left(D_{6}\right)$ and the shift vector to the form

$$
v=\frac{1}{\sqrt{2}}\left(\begin{array}{c}
1 \\
0^{5} \\
1 \\
0^{5}
\end{array}\right),
$$

This gives an enhanced symmetry group $S U(2)^{6}$ (studied in 40, 41, 42, 43, [44]). In the second theory, in order to reach these enhanced symmetry points we need to switch on background values of the fields $K_{i}^{\prime\left(\alpha^{\prime}\right)}$. These correspond to RR Wilson lines in the theory. Also some of the massless charged vector

\footnotetext{
${ }^{1}$ Note that we are using the basis in which the metric is $\left(\begin{array}{cc}0 & I_{2} \\ I_{2} & 0\end{array}\right)$ instead of $\left(\begin{array}{cc}-I_{2} & 0 \\ 0 & I_{2}\end{array}\right)$ used in ref. 44.
} 
bosons carry charge under the $\mathrm{U}(1)$ gauge fields $K_{\bar{\mu}}^{\prime\left(\alpha^{\prime}\right)}$ which originate in the $\mathrm{RR}$ sector. Thus we see again that the mechanism of symmetry enhancement is a non-perturbative effect in the second theory.

$\mathbf{N}=4$ Example B: In this set of examples we choose

$$
\left(\theta_{L}, \phi_{L}, \theta_{R}, \phi_{R}\right)=\left(\frac{4 \pi}{n}, 0,0,0\right), \quad n \geq 3
$$

This gives

$$
\left(\theta_{L}^{\prime}, \phi_{L}^{\prime}, \theta_{R}^{\prime}, \phi_{R}^{\prime}\right)=\left(\frac{2 \pi}{n},-\frac{2 \pi}{n}, \frac{2 \pi}{n},-\frac{2 \pi}{n}\right) .
$$

Physically, $\Omega$ given in eq.(4.14) corresponds to a rotation by $4 \pi / n$ in the $\left(6_{L}, 7_{L}\right)$ plane, whereas $\Omega^{\prime}$ given in eq.(4.15) corresponds to a left-right symmetric rotation by $2 \pi / n$ in the $(6,7)$ plane, and by $-2 \pi / n$ in the $(8,9)$ plane. For both the theories we choose the lattice shift in $\Gamma_{0}^{(2,2)}$ to be

$$
v=v^{\prime}=\left(\begin{array}{c}
1 / n \\
0 \\
0 \\
0
\end{array}\right)
$$

As in previous examples, adiabatic argument does not rule out the possibility of adding a term proportional to $(0,0,1 / n, 0)$ to $v$ and / or $v^{\prime}$, but the requirement of left-right level matching prevents the addition of such a vector. As in example A, both the theories have $N=4$ supersymmetry in four dimensions, with the first theory having $\left(0_{L}, 4_{R}\right)$ supersymmetry, and the second theory having $\left(2_{L}, 2_{R}\right)$ supersymmetry.

The analysis of the spectrum of massless $U(1)$ gauge fields can be carried out as follows. In the first theory, all the fields coming from the RR sector are projected out, so we only need to concentrate on the NS sector fields. Under the $\mathrm{U}(1)$ subgroup of $\mathrm{SO}(4,4)$ generated by $(\theta, 0,0,0)$ the vector representation of $\mathrm{SO}(4,4)$ decomposes as

$$
8_{v}=(+1) \oplus(-1) \oplus 6(0) .
$$

Thus of the eight vector fields $A_{\bar{\mu}}^{(a)}$, the six that are neutral under this $\mathrm{U}(1)$ subgroup survives the projection. Together with the four vector fields coming from the components $G_{i \bar{\mu}}, B_{i \bar{\mu}}$, these constitute ten $\mathrm{U}(1)$ vector fields. 
In the second theory, the relevant $\mathrm{U}(1)$ subgroup is generated by $(\theta,-\theta, \theta,-\theta)$. The various $\mathrm{SO}(4,4)$ representations have the following decomposition under this subgroup:

$$
\begin{aligned}
& 8_{v}=4(+1) \oplus 4(-1), \\
& 8_{c}=(+2) \oplus(-2) \oplus 6(0), \\
& 8_{s}=4(+1) \oplus 4(-1) .
\end{aligned}
$$

Thus of the possible vector fields $A_{\bar{\mu}}^{\prime(a)}, K_{\bar{\mu}}^{\prime\left(\alpha^{\prime}\right)}$, and those associated with the field strengths $D_{i \bar{\mu} \bar{\nu}}^{\prime(\alpha)}$, only six from $K_{\bar{\mu}}^{\prime\left(\alpha^{\prime}\right)}$ are neutral under this $\mathrm{SO}(2)$, and hence survives this projection. Together with the gauge field components $G_{i \bar{\mu}}^{\prime}, B_{i \bar{\mu}}^{\prime}$, this gives ten $\mathrm{U}(1)$ gauge fields altogether, in agreement with the calculation in the first theory.

As argued before, in this case the duality groups acting on the $\mathrm{S}$, T and $\mathrm{U}$ fields in both theories are given by $\Gamma_{0}(n)$. Thus the moduli space has the structure

$$
\left(\Gamma_{0}(n) \backslash S L(2, R) / U(1)\right) \times(G \backslash O(6,4) / O(6) \times O(4)),
$$

where

$$
G \cap O(2,2)=\Gamma_{0}(n) \times \Gamma_{0}(n) .
$$

The choice of the holonomy $\Omega$ is the same as that appears in some of the examples 44, however the choice of the lattice we have made is different from that chosen there. In particular the lattices studied there, which do lead to enhanced gauge symmetries for $n=3$ corresponding to $S O(5) \times S U(3)$ and $S U(3) \times S U(3)$, for $n=4$ corresponding to $S U(4) \times S U(2)$ and for $n=6$ corresponding to $S O(5) \times S U(2) \times S U(2)$, do not have a decomposition as $\Gamma^{(4,4)}+\Gamma^{(2,2)}$. However by turning on gauge field components $A_{i}^{(a)}(i=$ $4,5)$, and choosing appropriate shift vectors in the internal theory which correspond to turning on RR Wilson lines in the dual theory we can obtain all these models as special cases of the above dual pairs.2

\footnotetext{
${ }^{2}$ Another example considered in [44] with gauge group $G_{2}$ is likely to be dual to the non-geometric $\left(2_{L}, 2_{R}\right)$ compactification with left-right holonomy $S U(2)$ manifold for which we mod out by $(\pi,-\pi, 0,0)$ accompanied by the adiabatic shift $(1 / 2,0,0,0)$ and by $(0,0, \pi,-\pi)$ accompanied by the adiabatic shift $(0,1 / 2,0,0)$.
} 
$\mathbf{N}=2$ Example A: We shall now give an example of a self-dual $\mathrm{N}=2$ compactification. We choose,

$$
\left(\theta_{L}, \phi_{L}, \theta_{R}, \phi_{R}\right)=(2 \pi, 0, \pi,-\pi)
$$

This gives

$$
\left(\theta_{L}^{\prime}, \phi_{L}^{\prime}, \theta_{R}^{\prime}, \phi_{R}^{\prime}\right)=(2 \pi, 0, \pi,-\pi) .
$$

Also, the lattice with this symmetry is the original lattice $\Gamma_{0}^{(4,4)}$. This corresponds to $\langle M\rangle=\left\langle M^{\prime}\right\rangle=I_{8}$.

Physically, both $\Omega$ and $\Omega^{\prime}$ represent the transformation $(-1)^{F_{L}}$ together with an inversion of the right hand part of the lattice $\Gamma^{(4,4)}$ associated with $T^{4}$. We accompany this transformation with a lattice shift on $\Gamma_{0}^{(2,2)}$ of the form:

$$
v=v^{\prime}=\left(\begin{array}{c}
\frac{1}{2} \\
0 \\
0 \\
0
\end{array}\right) .
$$

Since the two theories are identical, we have here an example of a self-dual theory. Since under the duality transformation $S$ and $T$ gets exchanged, we see that this theory is invariant under the exchange $S \leftrightarrow T$.

The supersymmetry is completely broken in the left sector, and half broken in the right sector. Thus this theory has $\left(0_{L}, 2_{R}\right)$, i.e. $\mathrm{N}=2$ space-time supersymmetry. To calculate the massless spectrum in the theory, we study the decomposition of various representations of the $\mathrm{SO}(4,4)$ group under the $\mathrm{SO}(2)$ subgroup generated by $(2 \theta, 0, \theta,-\theta)$ :

$$
\begin{aligned}
& 8_{v}=(+2) \oplus(-2) \oplus 2(0) \oplus 2(+1) \oplus 2(-1), \\
& 8_{c}=(+2) \oplus(-2) \oplus 2(0) \oplus 2(+1) \oplus 2(-1), \\
& 8_{s}=2(+1) \oplus 2(-1) \oplus(+2) \oplus(-2) \oplus 2(0) .
\end{aligned}
$$

Since $\Omega$ corresponds to $\mathrm{SO}(2)$ rotation by $\pi$, we need to look for states that carry even $\mathrm{SO}(2)$ charge. Thus we get four gauge fields from $A_{\bar{\mu}}^{(a)}$, four from $K_{\bar{\mu}}^{\left(\alpha^{\prime}\right)}$, four $(=4 \times 2 / 2)$ field strengths from $D_{i \bar{\mu} \bar{\nu}}^{(\alpha)}$ and four more gauge fields from $G_{i \bar{\mu}}$ and $B_{i \bar{\mu}}$. This gives a total of 16 vector fields, which correspond to 15 vector multiplets.

Counting of scalars proceeds in a similar manner. First of all, the condition that the lattice associated with $T^{4}$ is invariant under inversion on 
the right completely fixes $\langle M\rangle$, so we do not get any scalar moduli fields from there. We get four scalars from $\psi^{(\alpha)}$, eight $(=4 \times 2)$ from $A_{i}^{(\alpha)}$, eight $(=4 \times 2)$ from $K_{i}^{\left(\alpha^{\prime}\right)}$, four from the field strengths $D_{i j \bar{\mu}}^{(\alpha)}$, four from $G_{i j}, B_{i j}$, and two from the dilaton-axion combination. This gives a total of 30 scalar fields, which form part of the 15 vector multiplets. Thus the theory does not contain any massless hypermultiplet.

The twisted sector in this theory for some values of the scalar moduli (for example the one corresponding to the moduli where in the $N=4$ example A gave $S U(2)^{2}$ enhanced gauge symmetry) will contain charged massless hypermultiplets. Thus we expect that the moduli space for vector multiplets receives quantum corrections. This is in accord with the fact that for this model the dilaton is in the vector multiplet.

$\mathbf{N}=2$ Example $\mathrm{B}$ : If we consider a $Z_{2} \times Z_{2}$ orbifold consisting of the modding outs we used in the $N=4$ example A and $N=2$ example A, where we use the adiabatic shift vectors to be on two different circles of $\Gamma_{0}^{(2,2)}$ we obtain another $N=2$ model. Now, however the model is not self-dual. The model and its dual receive their supersymmetry by $\left(0_{L}, 2_{R}\right)$ and thus have the dilaton in the vector multiplet. It is easy to see that generically the model has 7 vector multiplets and no hypermultiplets and that the dilaton is in a vector multiplet (compared to the previous example all the vector multiplets coming from the $\mathrm{RR}$ sector have been projected out by the second $Z_{2}$ ). For some values of the scalar moduli, for example the one corresponding to the moduli where in the $N=4$ example A gave $S U(2)^{2}$ enhanced gauge symmetry, this theory has perturbative non-abelian $S U(2)$ gauge symmetry with no matter. We thus expect there to be quantum corrections to the moduli geometry in accord with the fact that the dilaton is in a vector multiplet.

$\mathbf{N}=2$ Example $\mathbf{C}$ : Another example of a self-dual $\mathrm{N}=2$ model is obtained by modding out the theory by a $Z_{2} \times Z_{2}$ group generated by

$$
\begin{aligned}
\Omega_{1}=(2 \pi, 0,0,0), & v_{1}=\left(\begin{array}{c}
\frac{1}{2} \\
0 \\
0 \\
0
\end{array}\right), \\
\Omega_{2}=(\pi,-\pi, \pi,-\pi), & v_{2}=\left(\begin{array}{c}
0 \\
\frac{1}{2} \\
0 \\
0
\end{array}\right),
\end{aligned}
$$


The duality map exchanges $\Omega_{1}$ and $\Omega_{2}$. Thus the theory is self-dual provided we exchange the two circles of $T^{2}$. This corresponds to the exchange $S \leftrightarrow T$, $U \rightarrow-1 / U$. At a generic point in the moduli space the theory has three vector multiplets and four hypermultiplets. Perturbatively, enhanced SU(2) gauge symmetry occurs at points in the moduli space where the $N=4$ example A develops an enhanced $S U(2)^{2}$ gauge symmetry, but the structure of the moduli space near this point is corrected acoording to the results of Seiberg and Witten [50]. (The perturbative version of such corrections have been worked out in refs. 51, 52.) The $S \leftrightarrow T$ symmetry of the theory means that a similar structure of the theory must appear for large $T$ as well.

Beyond Adiabatic Argument: Even though the adiabatic argument seems to be powerful enough to suggest many new dual pairs, it has its limitation as many interesting cases and in particular models with $N=1$ supersymmetry cannot be constructed in this way. In this section we would like to abstract a general lesson from the validity of the adiabatic argument and suggest other ways of constructing dual pairs. This will open up our hands in constructing a much larger class of dual pairs.

One of the key checks of having a dual pair is the perturbative agreement of the massless spectra of the two models at generic points in the moduli space (special points may arise where perturbatively massless states in one model will have solitonic interpretation in the dual model). The adiabatic method of constructing dual pair already satisfies this for the following reason: We start with the massless modes in the higher dimensional theory (in our case $d=6)$ and take their momenta to be independent of the directions we are compactifying (in our case further compactification on $T^{2}$ ). Moreover we keep only the massless state invariant under the automorphism to be modded out by. Clearly the massless states we thus keep in the two theories are in one to one correspondence since we have an explicit isomorphism that relates the two theories and the corresponding automorphisms. Moreover the fact that the twisted sector is generically massive (as follows from the fact that we are going a fraction around a circle) implies that we have already accounted for all the massless states, and thus the two theories agree as far as the massless modes are concerned.

Note that from the point of view of the low dimensional observer, the states coming from the twisted sector are massive due to the Bogmol'nyi bound for the mass. It is thus natural to extend this principle also to the contribution to the Bogomol'nyi bound from the central charges arising from 
the internal $T^{4}$. In other words, if we mod out by transformations, all of which have shift vectors that generically have components along the central charges, then the corresponding twisted sector states are generically all massive and thus the previous argument for agreement between the massless states for the two theories will continue to hold. Using this idea we can construct dual models with any value of $N$ (excluding $N=7$ ). We will now illustrate this idea by constructing dual models with $N=1$ and $N=2$ supersymmetry in four dimensions as well as an $N=2$ model in six dimensions.

$\mathbf{N}=2$ Example D: We consider a $Z_{2} \times Z_{2}$ orbifold where we start from the $Z_{2}$ orbifold model of the $N=4$ example A. We mod out further by a $Z_{2}$ transformation which acts on the internal $T^{4}$ by

$$
\left(\theta_{L}^{\prime}, \phi_{L}^{\prime}, \theta_{R}^{\prime}, \phi_{R}^{\prime}\right)=(\pi, 0, \pi, 0)
$$

accompanied by a left-right symmetric half-vector shift on both $\theta$ and $\phi$ planes and a reflection on the common $T^{2}$. The dual to this second $Z_{2}$ according to Eq.(2.14) is given by,

$$
\left(\theta_{L}, \phi_{L}, \theta_{R}, \phi_{R}\right)=(\pi, 0, \pi, 0)
$$

accompanied by RR gauge transformations corresponding to the shifts we have introduced. This corresponds to turning on half units of flux of some of the RR gauge fields at the fixed points of this $Z_{2}$ transformation on $T^{4}$. It is easy to see that this theory has $N=2$ supersymmetry where in the primed theory it comes from $\left(1_{L}, 1_{R}\right)$ and in the unprimed theory it comes from $\left(0_{L}, 2_{R}\right)$. Note also that in the unprimed theory the fact that we get nothing from the twisted sector is very much analogous to a similar situation considered in ref. [25]. This model has generically 3 vector multiplets and 4 hypermultiplets. As far as vector moduli is concerned we can use the primed theory (where the dilaton is in the hypermultiplet) to compute the exact moduli space, and we see that it has moduli space $\left(\Gamma_{0}(2) \backslash S L(2, R) / U(1)\right)^{3}$, describing the $T$-moduli associated with the $\theta^{\prime}$-plane, the $\phi^{\prime}$-plane, and the torus $T^{2}$. This agrees with the classical moduli space for the unprimed theory. Thus there is no quantum correction in accord with the fact that there in the vector moduli there is never an enhanced gauge symmetry point. The hypermultiplet moduli can be computed from the unprimed theory which gives us the standard moduli space $G \backslash O(4,4) / O(4) \times O(4)$ describing the 
four-torus involving the $\theta$-plane and $T^{2}$. The discrete duality group $G$ is a subgroup of $S O(4,4 ; Z)$ which leaves the shift vector $v$ given in eq. (4.7) and the background RR flux invariant. This again agrees with the classical moduli of the primed theory. Thus that receives no corrections as well.

$\mathbf{N}=1$ Example: Let us now construct an $N=1$ example of dual pairs. We consider a $Z_{2} \times Z_{2}$ orbifold. One of the $Z_{2}$ 's is again the one considered in the self-dual $N=2$ Example A. The second $Z_{2}$ we consider is the same one considered in the last example, i.e. given by

$$
\left(\theta_{L}^{\prime}, \phi_{L}^{\prime}, \theta_{R}^{\prime}, \phi_{R}^{\prime}\right)=(\pi, 0, \pi, 0)
$$

accompanied by a left-right symmetric half-vector shift on both $\theta$ and $\phi$ planes and a reflection on the common $T^{2}$. This has the same holonomy dual but with RR fields turned on. This theory has $N=1$ supersymmetry coming from $\left(0_{L}, 1_{R}\right)$. Generically the theory has 9 chiral multiplets (including the dilaton) and 6 vector multiplets (four of the $U(1)$ gauge fields come from RR sector). The gauge symmetry is always abelian, consistent with the fact that we do not expect gaugino condensation with supersymmetry breaking.

6-dimensional Examples: Continuing in the same spirit, we can construct dual pairs of models in six dimensions. Consider, for example, the $N=2$ supersymmetric theory in six dimensions obtained by modding out the maximally supersymmetric $(N=4)$ theory by $\left(\theta_{L}, \phi_{L}, \theta_{R}, \phi_{R}\right)=(2 \pi, 0,0,0)$, together with a left-right symmetric half shift in the $\phi$ plane. This gives a $\left(0_{L}, 2_{R}\right)$ theory with $8 \mathrm{U}(1)$ gauge fields. Its dual is given by modding out the same theory by $\left(\theta_{L}^{\prime}, \phi_{L}^{\prime}, \theta_{R}^{\prime}, \phi_{R}^{\prime}\right)=(\pi,-\pi, \pi,-\pi)$, i.e. an inversion on the torus, together with a $\mathrm{RR}$ gauge transformation. This introduces a flux of RR gauge fields at the orbifold points and makes the twisted sector states massive. Thus we now get an $N=2$ supersymmetric $\left(1_{L}, 1_{R}\right)$ theory with 8 $\mathrm{U}(1)$ gauge fields. Construction of this dual pair is very similar in spirit to the construction of the dual of the CHL string discussed in ref. 25. As in [25], we expect this theory to have a solitonic string with half the tension of a fundamental string.

We would like to acknowledge the hospitality of the Aspen Center for Physics. We would like to thank C. Hull and N. Warner for discussion. The research of C. Vafa was partially supported by NSF grant PHY-92-18167. A. Sen would also like to acknowledge the hospitality of Queen Mary and Westfield College and The University of Wales, Swansea during the course of the work. 


\section{References}

[1] A. Font, L. Ibanez, D. Lust and F. Quevedo, Phys. Lett. B249 (1990) 35.

[2] S. Rey, Phys. Rev. D43 (1991) 526.

[3] S. Kalara and D. Nanopoulos, Phys. Lett. B267 (1991) 343.

[4] A. Sen, Nucl. Phys. B404 (1993) 109 hep-th/9207053; Phys. Lett. B303 (1993) 22 hep-th/9209016; Mod. Phys. Lett. A8 (1993) 2023 hep-th/9303057.

[5] J. Schwarz, preprints hep-th/9209125, hep-th/9307121, hepth/9411178, hep-th/9503127.

[6] J. Schwarz and A. Sen, Nucl. Phys. B411 (1994) 35 hep-th/9304154; Phys. Lett. B312 (1993) 105 hep-th/9305185.

[7] A. Sen, Int. J. Mod. Phys. A9 (1994) 3707 hep-th/9402002; Nucl. Phys. B434 (1995) 179 hep-th/9408083; preprint hep-th/9503057.

[8] J. Gauntlett and J. Harvey, preprint [hep-th/9407111.

[9] C. Hull and P. Townsend, Nucl. Phys. B438 (1995) 109 hepth/9410167].

[10] C. Vafa, unpublished.

[11] M. Duff, Nucl. Phys. B442 (1995) 47 hep-th/9501030.

[12] P. Townsend, Phys. Lett. B350 (1995) 184 [hep-th/9501068].

[13] E. Witten, preprint hep-th/9503124.

[14] A. Sen, preprint hep-th/9504027.

[15] J. Harvey and A. Strominger, preprint hep-th/9504047.

[16] C. Vafa and E. Witten, preprint hep-th/9505053.

[17] C. Hull and P. Townsend, preprint hep-th/9505073]. 
[18] S. Kachru and C. Vafa, preprint hep-th/9505105.

[19] S. Ferrara, J. Harvey, A. Strominger and C. Vafa, preprint hepth/9505162].

[20] V. Kaplunovsky, J. Louis and S. Theisen, preprint hep-th/9506110].

[21] A. Klemm, W. Lerche and P. Mayr, preprint hep-th/9506112.

[22] G. Papadopoulos and P. Townsend, preprint hep-th/9506150.

[23] A. Dabholkar, preprint hep-th/9506160.

[24] C. Hull, preprint hep-th/9506194.

[25] J. Schwarz and A. Sen, preprint hep-th/9507027.

[26] I. Antoniadis, E. Gava, K. Narain and T. Taylor, preprint hepth/9507115].

[27] C. Vafa and E. Witten, preprint [hep-th/9507050].

[28] E. Witten, preprint hep-th/9507121.

[29] J. Harvey, D. Lowe and A. Strominger, preprint hep-th/9507168.

[30] C. Montonen and D. Olive, Phys. Lett. B72 (1977) 117;

P. Goddard, J. Nyuts and D. Olive, Nucl. Phys. B125 (1977) 1.

[31] H. Osborn, Phys. Lett. B83 (1979) 321.

[32] A. Sen, Phys. Lett. B329 (1994) 217 hep-th/9402032].

[33] C. Vafa and E. Witten, Nucl. Phys. B431 (1994) 3 hep-th/9408074.

[34] L. Girardello, A. Giveon, M. Porrati and A. Zaffaroni, Phys. Lett. B334 (1994) 331 [hep-th/9406128]; preprint hep-th/9502057.

[35] M. Porrati, preprint hep-th/9505187.

[36] A. Schellekens, unpublished.

[37] S. Chaudhuri, G. Hockney and J. Lykken, preprint hep-th/9505054. 
[38] S. Chaudhuri and J. Polchinski, preprint hep-th/9506048].

[39] P. Aspinwall, Lectures at the Trieste conference on 'S-duality and Mirror Symmetry', June 5-9, 1995.

[40] R. Blum, L. Dolan and P. Goddard, Nucl. Phys. B289 (1987) 364.

[41] H. Kawai, D. Lewellen and S.H. Tye, Phys. Lett. 191B (1987) 63.

[42] W. Lerche, B.E.W. Nilsson and A.N. Schellekens, Nucl. Phys. B294 (1987) 136.

[43] S. Ferrara and C. Kounnas, Nucl. Phys. B328 (1989) 406.

[44] L. Dixon, V. Kaplunovsky and C. Vafa, Nucl. Phys. B294 (1987) 43.

[45] J. Dai, R. Leigh and J. Polchinski, Mod. Phys. Lett. A4 (1989) 2073;

R. Leigh, Mod. Phys. Lett. A4 (1989) 2767;

G. Pradisi and A. Sagnotti, preprint [hep-th/9211084].

[46] E. Cremmer and B. Julia, Phys. Lett. 80B (1978) 48; Nucl. Phys. B159 (1979) 141.

[47] J. Schwarz, Phys. Lett. 95B (1980) 219.

[48] Y. Tanii, Phys. Lett. B145 (1984) 197.

[49] C. Vafa, Mod. Phys. Lett. A4 (1989) 1615.

[50] N. Seiberg and E. Witten, Nucl. Phys. B426 (1994) 19 hep-th/9407087; Nucl. Phys. B431 (1994) 484 hep-th/9408099].

[51] B. De Witt, V. Kaplunovsky, J. Louis and D. Lust, preprint hepth/9504006].

[52] I. Antoniadis, S. Ferrara, E. Gava, K. Narain and T. Taylor, preprint hep-th/9504034. 